\title{
Détection directe et caractérisation des planètes extrasolaires
}

\author{
Alexis CARLOTTI \\ Université de Princeton, Département d'ingénierie mécanique et aérospatiale \\ acarlott@princeton.edu
}

À ce jour, 725 exoplanètes ont été détectées et 29 ont déjà été observées directement [1]. Ceci s'explique d'une part par la nature de ces objets : une planète âgée ne fait que refléter une maigre fraction de la lumière de son étoile, et les deux sont séparées par une courte distance angulaire. D'autre part nos moyens d'imagerie sont limités par la diffraction, la qualité des optiques, et la turbulence atmosphérique. L'optique adaptative et les télescopes spatiaux permettent de s'affranchir en partie de ces deux dernières contraintes. Pour se jouer de la diffraction, des techniques dites coronographiques seront employées d'ici 1 à 2 ans sur des télescopes au sol, permettant I'observation de planètes semblables à Jupiter. D'ici à 2020, deux télescopes spatiaux en seront également dotés et observeront des planètes de quelques masses terrestres. Enfin, de futurs télescopes géants actuellement à l'étude seront peut-être en mesure de faire de même pour des planètes de type terrestre.

\section{Un problème d'imagerie à haut contraste et haute résolution angulaire}

\section{Observations pionnières}

Certaines étoiles étant âgées de quelques millions d'années seulement, leurs planètes sont encore assez jeunes pour rayonner dans l'infrarouge une fraction de leur énergie de formation. Cet éclat propre rend leur observation plus facile que celle de planètes évoluées, et explique principalement comment ontété obtenues les premières images directes de systèmes planétaires. Deux cas méritent d'être mentionnés en particulier: HR8799 (figure 1), observée depuis 2004 par Marois et al. et Beta Pictoris observée depuis 2003 par Lagrange et al. Les télescopes utilisés comptent parmi les plus grands au monde (entre 8 et 10 mètres de diamètre). Les plus récentes techniques d'optique adaptive ont été utilisées ainsi que des techniques d'imagerie différentielle /soustractions d'images enregistrées à différentes longueurs d'onde, différentes polarisations ou pour différentes orientations du télescope). Soummer et al. ont pu retrouver trois des quatre planètes du système HR8799 dans des images prises par le télescope spatial Hubble dès 1998 et grâce à d'autres techniques avancées

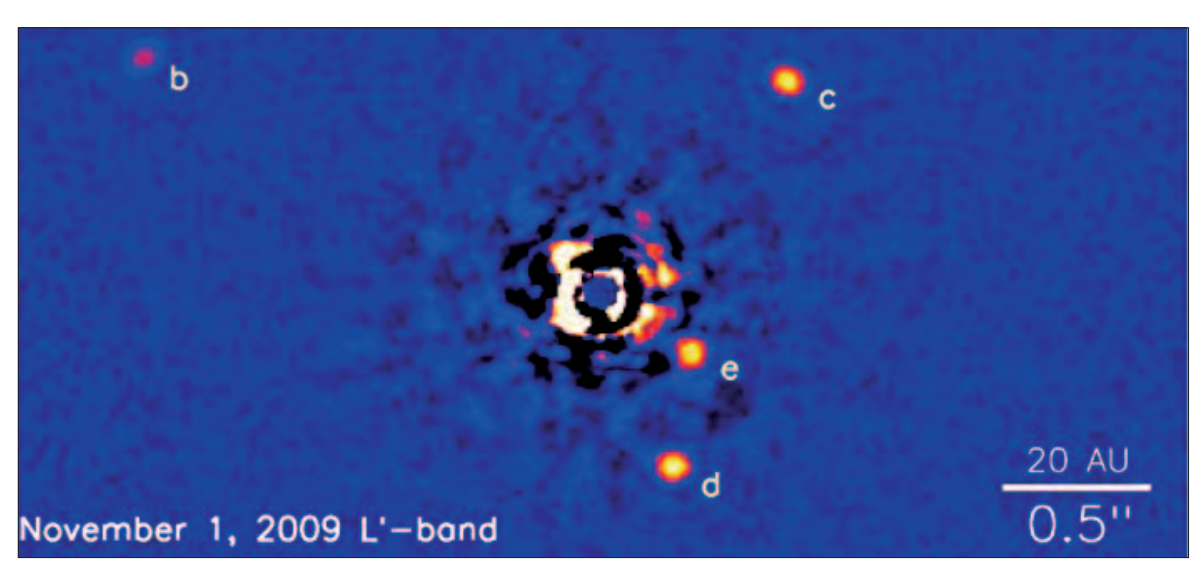

Figure 1. Observation de l'étoile HR8799 par Marois. On distingue 4 planètes autour de l'étoile. Seuls des résidus de la lumière de l'étoile apparaissent sur cette image. L'essentiel en a été retiré par imagerie différentielle.

de traitement d'image. Des planètes plus âgées ontégalementété observées, mais uniquement car la distance qui les sépare de leur étoile est excessivement grande. Le télescope Hubble a ainsi été utilisé par Kalas et al. pour détecter une planète âgée de 220 Ma mais distante de 115 fois la distance Terre-Soleil de son étoile, Fomalhaut.

Observer des planètes plus communes

Si à l'aide d'un simple télescope on voulait observer dans le visible une planète semblable à la Terre et se trouvantà 10 pc de nous ( 1 pcou parsec estégal à 3,2615 années-lumière), son miroir devrait mesurer plus d'un kilomètre de diamètre pour que la lumière de la planète ait la même intensité que le halo de diffraction entourant l'étoile. Le problème consiste donc à se débarrasser de la lumière diffractée de l'étoile, et ce sans bloquer la lumière de la planète. Trois types d'instruments permettent d'y arriver : les interféromètres multi-ouvertures, les occulteurs externes et les coronographes. La mise en œuvre des deux premières techniques repose sur I'utilisation de plusieurs télescopes ou de 
plusieurs satellites, tandis que la dernière ne repose que sur l'utilisation d'un seul télescope, qu'il soit au sol ou dans l'espace.

Un coronographe peut avoir deux objectifs différents : soit celui d'annuler totalement ou d'atténuer suffisamment la lumière arrivant sur l'axe, et donc son halo de diffraction, soit celui de changer dans le plan image la répartition de la lumière diffractée pour l'atténuer dans une zone où une planète pourrait se situer. Dans le premier cas on utilisera principalement des masques focaux ou des interféromètres mono-pupilles, tandis que dans le second on aura recours à des masques pupilles ou à des anamorphoses de pupilles. II est souvent possible, voire souhaitable, de combiner plusieurs de ces techniques.

\section{La grande famille des coronographes}

Jusqu'au début des années 1930, personne n'avait réussi à observer les abords peu lumineux du Soleil sans que la Lune ne vienne l'éclipser totalement, un phénomène qui ne se produit qu'une fois par an sur Terre, et qui ne dure guère plus d'une minute. L'astronome Bernard Lyot s'attaqua au problème et présenta en 1932 son coronographe ([2] et figure 2). Dans le plan image formé par une première lentille, un masque réfléchissant est placé pour renvoyer la lumière du disque. Si l'on reforme un nouveau plan pupille on s'aperçoit qu'une frange brillante entoure le bord de l'ouverture. Celle-ci est due à la diffraction du masque, et peut être éliminée en plaçant un diaphragme dans ce plan pupille intermédiaire. Même si les performances du coronographe de Lyot classique ne permettent pas de détecter des exoplanètes, la plupart des coronographes modernes sont basés sur cette architecture initiale, et c'est l'utilisation de différents masques pupilles et focaux qui les en différencie.

\section{Des apodiseurs pour remodeler la figure de diffraction}

Comme la réponse de l'instrument est donnée par la transformée de Fourier de la fonction pupille, modifier la transmission

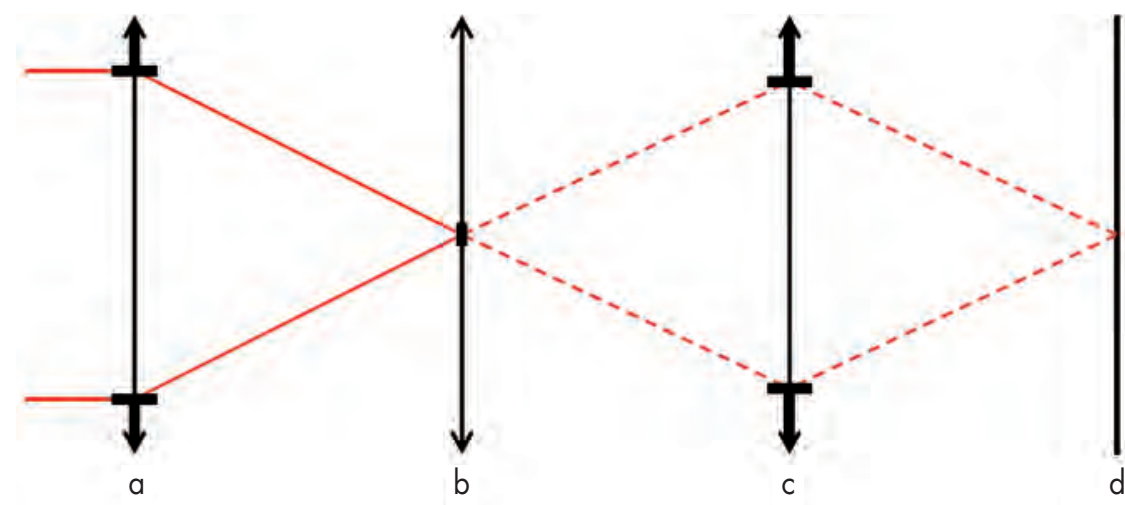

Figure 2. Schéma du coronographe de Lyot. Le plan a est celui de la pupille du télescope. Le plan b est un plan focal dans lequel est placé le masque de Lyot. Le plan c, ou plan de Lyot, est un nouveau plan pupille dans lequel un diaphragme (Lyot stop) sert à bloquer la lumière diffractée par le masque. Le plan $d$ est le plan focal final dans lequel a lieu l'observation.

de celle-ci modifie également le halo de diffraction qui entoure l'étoile. C'est tout I'objet de cette technique, dont le nom fut choisi par Jacquinot en 1954. Plusieurs approches existent pour déterminer cette transmission, si possible de la manière la plus optimale.

On peut par exemple vouloir concentrer le plus de lumière possible dans une petite zone au centre du plan focal. Cette technique optimise ainsi la réjection du coronographe de lyot si, comme l'ont montré Aime et al. en 2002 [3], les transmissions choisies font partie de la famille des fonctions sphéroïdales prolates étudiées auparavant par Slepian et Frieden.

La fabrication d'un tel masque peut se faire de différentes manières. On peut par exemple déposer une couche métallique d'épaisseur variable sur un substrat de verre, ou utiliser un substrat spécial dont on aura changé l'opacité en le bombardant avec un faisceau d'électrons. Ces deux techniques sont néanmoins assez chromatiques et ne garantissent pas une précision suffisante quant à la transmission obtenue. On peut ainsi plutôt choisir d'approximer cette transmission continue par une distribution de micro-points opaques imprimés sur un substrat par photolithographie. Cette technique a été retenue pour la fabrication des apodiseurs qu'utiliseront les instruments SPHERE et GPI respectivement installés aux foyers du Very Large Telescope (VLT) et du Gemini South Telescope, tous les deux installés au Chili, et d'un diamètre d'environ 8 mètres. Cesins- truments auront pour objectifl'observation dans l'infrarouge proche de planètes joviennes relativement peu âgées. Ils devraient voir leur première lumière au cours de l'année 2013. Afin de corriger les imperfections de leurs optiques, ces deux coronographes seront associés à des systèmes d'optique adaptative utilisant des miroirs déformables dotés d'un grand nombre d'actuateurs (jusqu'à 4000). L'utilisation de ces systèmes correcteurs de front d'onde est indispensable pour retrouver, dans une zone limitée du plan image, les performances théoriques des instruments précédents (figure 3).

Ces apodiseurs en absorption réduisent inévitablement la transmission de l'instrument. Comme l'a montré Guyon en 2003, il est également possible d'utiliser des miroirs (ou lentilles) asphériques afin de procéder à une anamorphose de la répartition d'intensité dans le plan pupille, et donc une apodisation, et ce sans perte de transmission. De plus, ces miroirs créent un effet de zoom qui permet I'accès à une plus haute résolution. En pratique la forme des miroirs à utiliser est caractérisée par une très forte courbure au bord de la pupille, ce qui rend leur fabrication délicate. Une solution consiste à obtenir la majeure partie de l'apodisation désirée via ce système, et à la compléter grâce à un apodiseur en absorption. Un instrument basé sur ce système, et lui aussi complété par un système d'optique adaptative extrême, est en cours d'assemblage au foyer du télescope Subaru, 

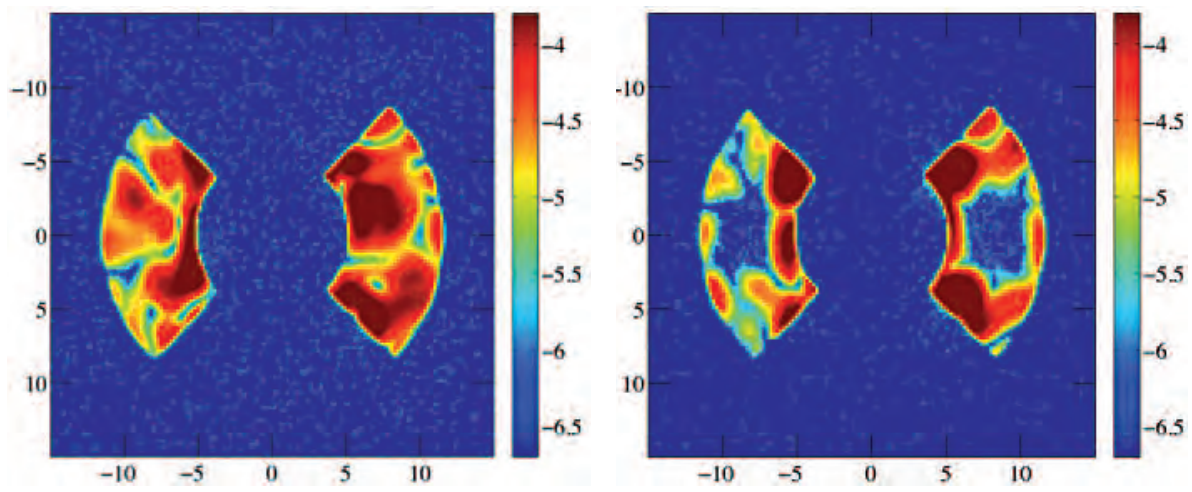

Figure 3. Images des abords de l'étoile (en échelle logarithmique) respectivement avant (à gauche) et après (à droite) correction du front d'onde par le système d'optique adaptative. Les deux zones en ailes de papillons sont celles dans lesquelles la lumière est transmise par le masque focal. À gauche le contraste moyen est de l'ordre de $10^{-4}$, tandis qu'à droite un contraste de l'ordre de $10^{-7}$ est créé par l'optique adaptative dans deux champs plus restreints.
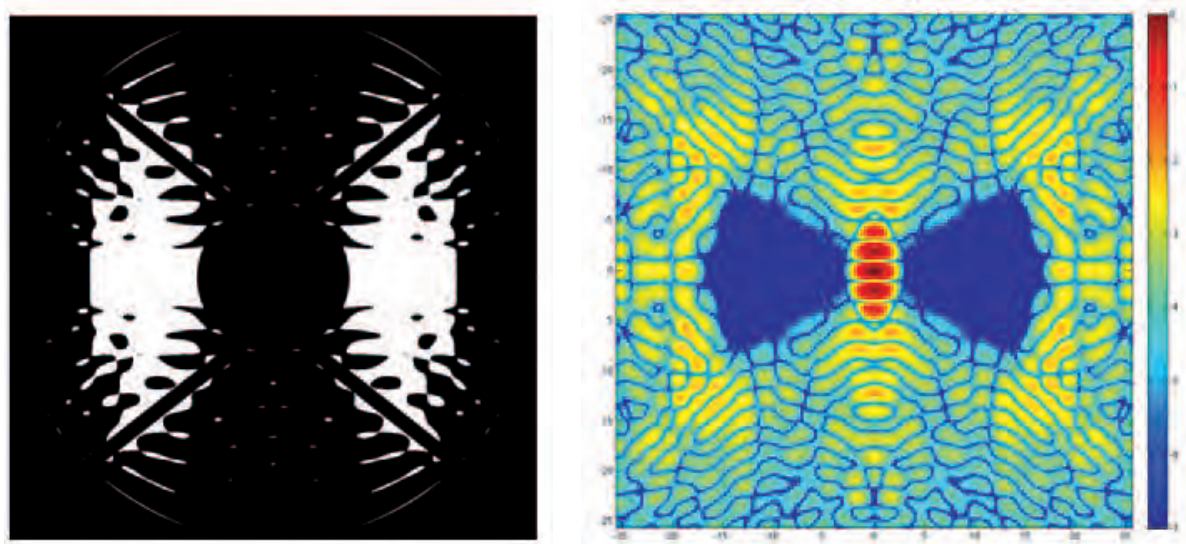

Figure 4. À gauche, plan pupille ré-imagé après la pose du masque apodiseur. Dans ce cas particulier ce masque a été optimisé pour la pupille du télescope Subaru. À droite, réponse de l'instrument (en échelle logarithmique, et en l'absence d'aberrations optiques). L'essentiel de la lumière est concentré au centre de l'image et deux zones de haut contraste (en bleu foncé) sont créées de part et d'autre. Le contraste théorique y est de $10^{-7}$

installé à Hawaii et d'un diamètre d'environ 8 mètres.

Une autre forme d'optimisation, proposée par Spergel, Kasdin et Vanderbei entre 2000 et 2003, consiste à maximiser la transmission du masque tout en exigeant de l'amplitude de l'onde qu'elle soit maintenue sous une certaine limite dans certaines régions du plan focal. D'abord réalisées en une seule dimension, elles ont récemment été généralisées à deux dimensions par Carlotti et al. [4]. Ces optimisations numériques débouchent sur des distributions quasi-binaires, qui peuvent être approximées à des distributions binaires dès lors que la pupille est décrite sur un nombre suffisant de points d'échan- tillonnage. L'intérêt principal de ces masques est qu'ils peuvent être optimisés pour n'importe quel type de télescope, par exemple à miroir segmenté et à obstruction centrale (figure 4). De plus, s'ils sont structurellement connectés, ils peuvent être réalisés en utilisant une fine feuille de métal dont certaines régions auront été découpées. Ce type d'apodiseur n'entraîne ainsi aucune erreur de front d'onde supplémentaire, ni aucun effet chromatique. Dans le cas où ils ne sont pas structurellement connectés, ils peuvent être réalisés par photolithographie, de la même manière que les apodiseurs micropoints. Des apodiseurs binaires seront utilisés par le coronographe qui équipera le

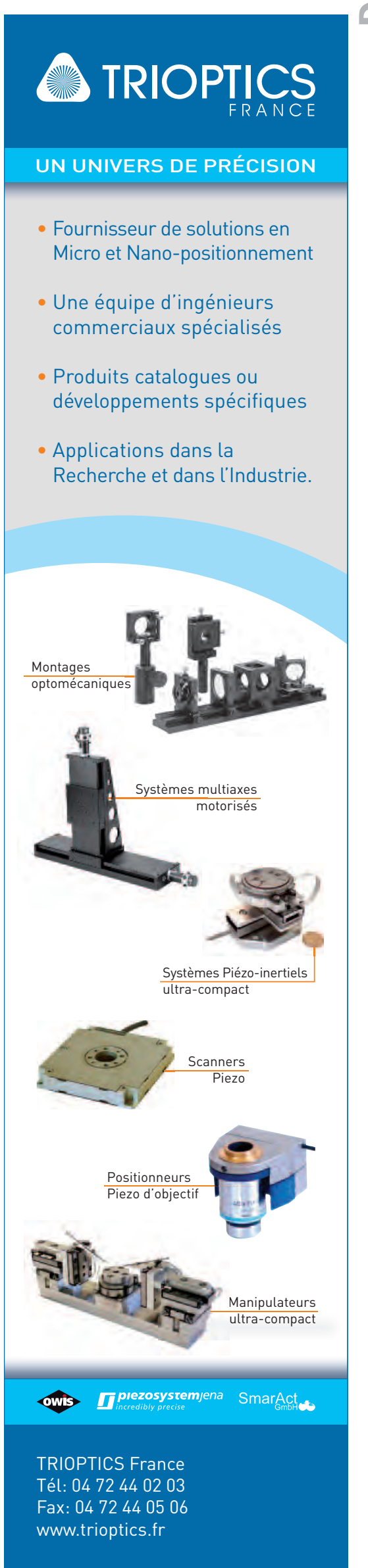

$\infty$
2
$a$
0
.0
$\frac{0}{5}$
$\frac{0}{0}$
$\frac{0}{0}$
$\frac{0}{2}$ 
télescope spatial SPICA dont le lancement est prévu en 2018. Ce télescope de 4 mètres de diamètre effectuera la caractérisation de planètes de type jovien dans des longueurs d'onde uniquement accessibles depuis l'espace (2,5 à 28 microns). Il est prévu qu'il soit le premier télescope spatial doté d'un miroir déformable.

\section{Annuler entièrement la lumière de l'étoile}

Le coronographe interférentiel achromatique, proposé en 1996 par Gay et Rabbia, fut l'un des premiers concepts coronographiques modernes. Un faisceau collimaté en provenance du télescope est injecté dans un interféromètre de Mach-Zehnder dans I'un des bras duquel est placé un œil de chat pour retourner le plan pupille de $180^{\circ}$. Ainsi, dans le plan focal reformé dans la voie de sortie destructive de l'instrument, l'étoile se trouve annulée tandis que l'image de la planète apparaît dédoublée. Cet instrument permet de sonder les abords les plus proches de l'étoile, et il est de fait particulièrement sensible à sa taille angulaire. Une solution possible consiste à apodiser le faisceau d'entrée. La lumière de l'étoile peut aussi être annulée en utilisant un interféromètre de type Sagnac à 2 ou 3 dimensions commel'ont suggéré Tavrov et al. Contrairement au Mach-Zehnder, cette configuration a l'avantage de posséder des chemins optiques naturellement équilibrés.

Le masque de Lyot, opaque, peut être remplacé par un masque de phase, créant un déphasage de rentre sa partie interne et sa partie externe. Associée à une apodisation prolate, cette technique, proposée par Roddier en 1997, permet d'annuler par interférence la totalité de la lumière de l'étoile. Comme l'a montré Rouan en 2000, le déphasage de $\pi$ peut aussi être induit entre les quadrants pairs et impairs du plan focal [5]. Ce masque à quatre quadrants possède un avantage sur le précédent : la précision avec laquelle le diamètre du disque déphasant est obtenu a une grande influence sur l'atténuation créée par le masque. En revanche la symétrie circulaire du masque de Roddier fait défaut au masque à quatre quadrants : la lumière de la planète est atténuée lorsqu'elle se trouve à cheval entre deux quadrants.
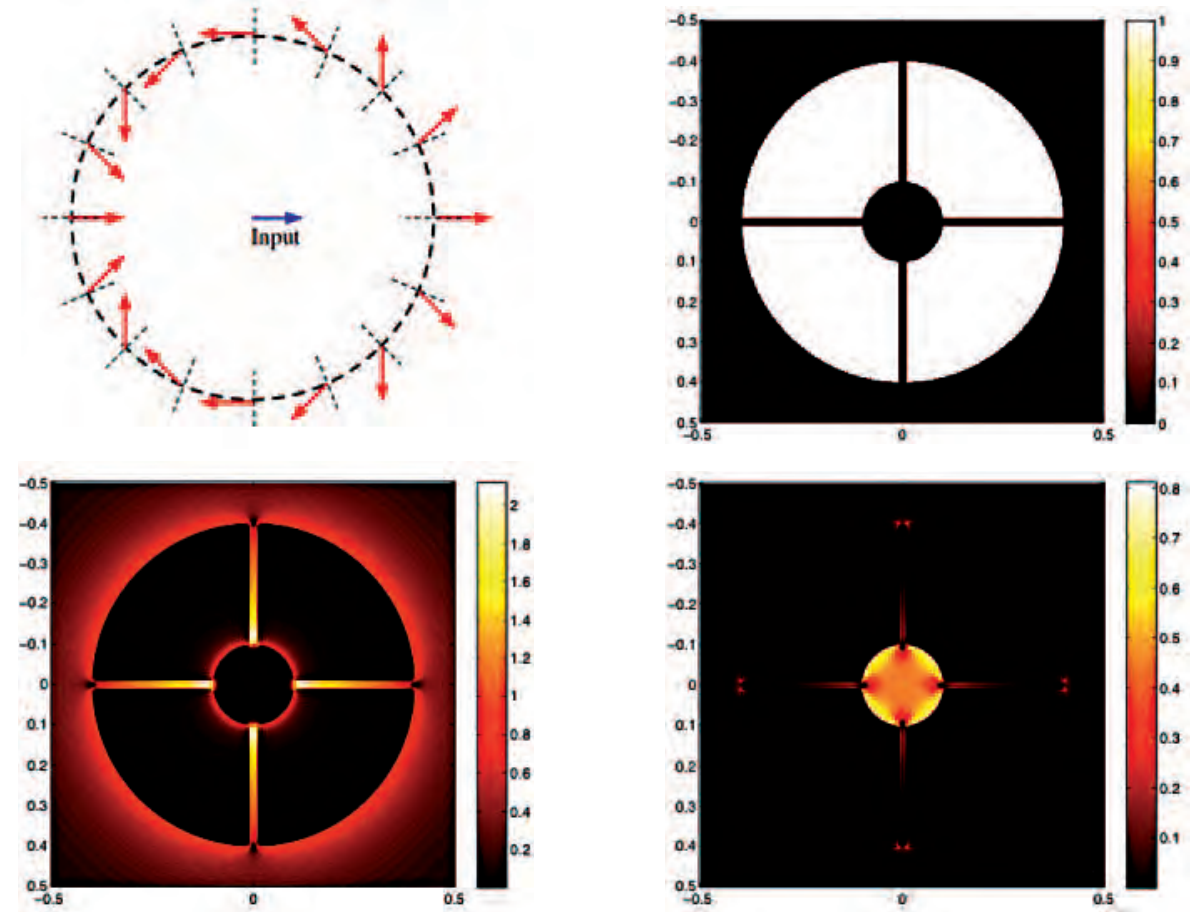

Figure 5. En haut, à gauche, schéma de l'effet d'un vortex optique de charge topologique 2. L'onde initiale est polarisée linéairement, et le masque provoque une rotation du vecteur de polarisation d'un angle double de celui de l'azimut du point considéré sur le masque. En haut, à droite, pupille à obstruction centrale utilisée dans le reste de la simulation. En bas, à gauche, simulation de la distribution d'intensité dans le premier plan de Lyot. La lumière est principalement rejetée en dehors de la pupille, mais l'obstruction centrale en rejette égalementà l'intérieur. En bas, à droite, simulation de la distribution d'intensité dans le second plan de Lyot. Un Lyot stop peut alors supprimer la quasi-totalité de la lumière.

Des prototypes de masques à quatre quadrants ont été testés dans les laboratoires de l'observatoire de Paris, puis au foyer du VLT. L'instrument SPHERE qui sera bientôt installé au VLT comportera plusieurs masques de ce type, chacun optimisé pour une longueur d'onde spécifique. Plusieurs masques à quatre quadrants seront aussi utilisés dans l'instrument MIRI qui équipera le télescope spatial JWST, d'un diamètre de 6,5 mètres et dont le lancement est prévu pour 2018. En 2005, Mawet proposa de déphaser la lumière d'un angle proportionnel à l'azimut d'un point considéré sur la surface du masque ([6] et figure 5) età un paramètre appelé charge topologique. Ce masque offre une atténuation de la lumière similaire à celle créée par le masque à quatre quadrants. Leur étude par l'optique de Fourier révèle qu'un masque à quatre quadrants peutêtre vu comme le résultat d'une somme infinie de masques vortex de charges topologiques croissantes. La principale différence entre ces deux masques est que, comme la transition de phase se fait de manière continue dans le cas du masque vortex, la lumière de la planète n'est pas atténuée le long des axes comme c'est le cas avec le masque à quatre quadrants.

Un prototype de masque vortex a permis d'observer au foyer du télescope Palomar les planètes externes du système HR8799. Ce test a néanmoins été fait sur une portion réduite de la pupille. En effet, alors que tous nos grands télescopes possèdent une obstruction centrale, les masques de phase de Roddier, à quatre quadrants et vortex ne fonctionnent de manière optimale qu' avec destélescopes sans obstruction centrale. Mawet a néanmoins démontré que deux masques vortex de sens de rotation opposés, placés dans deux plans focaux produits successivement permettent de minimiser l'impact de I'obstruction centrale (figure 5).

D'un point de vue pratique, la différence de phase que ces masques doivent introduire peut être induite en faisant varier 
l'épaisseur du substrat. Dans ce cas la lumière de l'étoile n'est alors annulée que dans une étroite bande de longueur d'onde. II est possible de reformer plusieurs plans focaux et de placer dans chacun d'eux un masque dédié à une seule longueur d'onde. Une phase faiblement chromatique peut en outre être introduite en utilisant un réseau de diffraction d'ordre zéro légalement appelé réseau sublambda, car sa finesse est plus petite que la longueur d'onde). Enfin, il est possible d'utiliser un film de cristaux liquides afin de manipuler dans le plan focal la polarisation, et donc la phase de l'onde.

\section{Les télescopes de demain}

Plusieurs très grands télescopes sont actuellement à l'étude. L'Europe a ainsi décidé la construction d'un télescope au sol de près de 40 mètres composé d'environ 1000 segments dont l'orientation pourra être individuellement ajustée. Les
États-Unis ont en projet un télescope au sol de 30 mètres basé sur une architecture similaire. Ces deux projets verront probablement leur première lumière au cours des années 2020. À plus long terme on envisage aussi le lancement d'un futur télescope spatial, d'un diamètre de 8, voire de 16 mètres. Là encore un miroir segmenté sera très probablement utilisé.

Chacun de ces projets cherchera, entre autres, à observer et caractériser des exoplanètes. La préparation des instruments coronographiques qui les équiperont a déjà commencé. Les plus hautes résolutions que ces télescopes offriront, ainsi que les plus grands nombres de photons qu'ils collecteront, permettront d'améliorer les performances de nos coronographes, et donc d'étudier des planètes à la fois plus petites que Jupiter, et plus proches de leur étoile, jusqu' à rendre possible la découverte et l'étude de planètes semblables à la Terre, et d'éventuelles formes de vie qu'elles pourraient abriter.

\section{Références}

[1] http://exoplanet.eu/catalog.php

[2] Lyot, B, "Étude de la couronne solaire en dehors des éclipses", Zeitschrift fur Astrophysik, 5, 73 (1932)

[3] Aime, C., Soummer, R., Ferrari, A., "Total coronagraphic extinction of rectangular apertures using linear prolate apodizations", Astronomy \& Astrophysics, 389, 334-344 (2002)

[4] Carlotti, A., Vanderbei, R., Kasdin, N.J., "Optimal pupil apodizations of arbitrary apertures for highcontrast imaging", Optics Express, 19, 26796-26809 (2011).

[5] Rouan, D., Riaud, P., Boccaletti, A., Clénet, Y., Labeyrie, A., "The Four-Quadrant Phase-Mask Coronagraph. I. Principle", Publications of the Astronomical Society of the Pacific, 112, 1479-1486 (2000)

[6] Mawet, D., Riaud, P., Absil, O., Surdej, J., "Annular Groove Phase Mask Coronagraph", The Astrophysical Journal, 633, 1191 (2005)

\section{Changing the Game}

\section{ORCA-Flash4.0}

Hamamatsu présente la première caméra scientifique SCMOS 2nde génération. Cette technologie permet d'atteindre les performances de toutes les caméras de première génération (CCD, EMCCD et CMOS).

Combinant un très faible bruit et un rendement quantique très élevé, I'ORCA-Flash 4.0 a une très grande sensibilité, une grande dynamique et une cadence de 100 images par seconde, tout cela en acquisition directe sur disque dur.

L'ORCA-Flash 4.0 est un véritable concentré de notre savoir-faire et elle va réellement changer les règles du jeu de l'imagerie scientifique.

Pour plus d'informations: www.hamamatsucameras.com/flash4

Applications:

- Electroluminescence sur cellules solaires

- Inspection de semiconducteurs (TSV, MEMS, Wafers)

- Imagerie de scintillateur (rayons $X$, microscopie électronique)

- Microscopie super-résolution

- Suivi de molécules uniques

- et toutes celles utilisant dec CCD bas bruits..
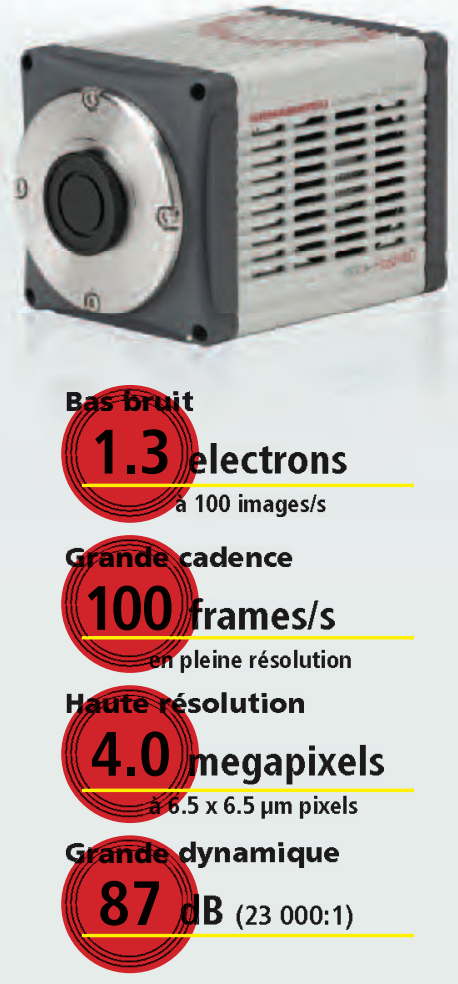